Key literature was assessed around a number of themes:

- Alcohol concentration in breast milk and time to elimination

- Level of potential alcohol exposure in the breastfeeding infant

- Effects on infant feeding, sleep and neurodevelopment

Methods A research request was made through the HSE Library Service to source relevant literature. Keywords in the search strategy included 'breastfeeding', 'breast milk', 'alcohol', 'ethanol', 'infant', 'paediatric'. Multiple databases were searched: Scopus, Pubmed, Embase, Web of Science, Cochrane, Cinahl, PsycINFO, Science Direct, and Clinical Key.

Titles and abstracts were assessed against pre-specified inclusion criteria. Full-text articles for studies meeting the inclusion criteria were retrieved for in-depth review. CASP checklists were used to assess study quality.

Results 3 systematic reviews and 27 observational studies were identified.

Alcohol level peaks in breast milk about 30-60 minutes post-ingestion.

2 hours required, on average, to metabolise 1 standard drink. Breast milk will contain alcohol until this time has elapsed.

If an infant were fed at peak alcohol concentration it will receive only about $3 \%$ of the maternal 'dose'.

No significant effect on the amount the infant drinks at the breast. It may result in more interrupted infant sleep. No effect on neurodevelopment.

Key messages Avoid alcohol in the first month postpartum as feeding is very frequent and it takes time to establish a routine.

For women breastfeeding beyond 1 month:

- Continue to adhere to guidance on low-risk alcohol consumption.

- Feed your baby before having a drink.

- Express milk before drinking alcohol. This will allow you to feed your baby if they need feeding before you are ready. Conclusion This review provides an important update to HSE advice on alcohol use and breastfeeding. Our findings have since been incorporated into new user-friendly guidance, available at askaboutalcohol.ie, breastfeeding.ie and the recently launched mychild.ie. The information presented is consistent throughout to ensure robust, clear and transparent advice.

\section{P303 A MULTICENTRE HEALTH PROMOTION STUDY, EXAMINING PARENTAL KNOWLEDGE OF AND REDUCING EXPOSURE TO PASSIVE SMOKING (REPS)}

\footnotetext{
${ }^{1}$ Sheena Coyne*, 2,3 Michaela Pentony, ${ }^{1}$ Juliette Lucey, ${ }^{1}$ Navdeep Kaur Brar, ${ }^{1}$ Bazlin Ramly. ${ }^{1}$ University Hospital Waterford, Waterford, Ireland; ${ }^{2}$ Our Lady of Lourdes Hospital Drogheda, Louth, Ireland; ${ }^{3}$ Temple Street Children's University Hospital, Dublin, Ireland
}

\subsection{6/archdischild-2019-epa.652}

The harmful effects of environmental tobacco smoke on the paediatric population is well documented in the literature. The aim of this study is to examine the existing parental knowledge between passive smoking and increased rates of paediatric hospitalisation and to provide an intervention in the form of referral to a smoking cessation programme.

A pilot study was conducted in a tertiary paediatric centre. This prospective cohort study recruited thirty-six paediatric patients presenting with respiratory tract infections. Consenting families were asked seven questions regarding their smoking habits. Following completion of the pilot study a further fifty patients from two level two paediatric centres were identified. In the level two centres focus was narrowed to children directly affected by passive smoking. Parents accepting referral to a twelve-week smoking cessation programme are currently being followed up over a six-month period. In the case of parents declining a referral to smoking cessation, a reason for refusal was also sought.

From the pilot, although most claimed a desire to quit smoking, acceptance of referral to the smoking cessation programme was poor. Results from the level two centres where the service is offered on site reflected better uptake with $52 \%$ (26/50) accepting a referral to smoking cessation services. Over the three centres only $69 \%$ of parents claimed an awareness of the harmful effects of environment tobacco smoke with a wide variation between different geographical areas. Results comparing the three centres are demonstrated in the table below.

\begin{tabular}{llll} 
Abstract P303 & Table 1 & & \\
\hline & $\begin{array}{l}\text { Site A } \\
\text { Tertiary Centre }\end{array}$ & $\begin{array}{l}\text { Site B } \\
\text { Level two centre }\end{array}$ & $\begin{array}{l}\text { Site C } \\
\text { Level two centre }\end{array}$ \\
\hline No. of patients & 36 & 25 & 25 \\
Smokers & 14 & 25 & 25 \\
Desire to quit & $71 \%(10 / 14)$ & $80 \%(20 / 25)$ & $92 \%(23 / 25)$ \\
Referred & $28 \%(4 / 14)$ & $64 \%(16 / 25)$ & $40 \%(10 / 25)$ \\
Awareness & $86 \%(31 / 36)$ & $44 \%(11 / 25)$ & $68 \%(17 / 25)$ \\
\hline
\end{tabular}

Preliminary results from smoking cessation team showed that $50 \%(13 / 26)$ of those referred from the level two centres attended their cessation appointments. 11\% (3/26) changed their minds once contacted by the smoking cessation team and the remainder were uncontactable or have not yet made an appointment. $23 \%(6 / 26)$ were smoke free for the last fourteen days at the time of follow up.

Our data suggests scope to improve education of parents on the harmful effects of environmental tobacco smoke. We also suggest that referral to smoking cessation services routinely be offered to parents of children exposed to environmental tobacco smoke as a cost-effective health promotion strategy. Recruitment is ongoing.

\section{P304 THE CASE OF PERTUSSIS IN NEWBORNS AND INFANTS: THE EPIDEMIOLOGY THAT 'COUNTS' IN VACCINATION CHOICES}

${ }^{1}$ Federico Marchetti, ${ }^{2}$ Claudia Guiducci, ${ }^{1}$ Lorenzo Mambelli*. ${ }^{1}$ Department of Pediatrics, S. Maria delle Croci Hospital, Ravenna, Ravenna, Italy; ${ }^{2}$ University Hospital Arcispedale Sant'Anna, Ferrara, Italy

\subsection{6/archdischild-2019-epa.653}

From June to August 2018, 6 infants with pertussis aged between 1 and 9 months ( 4 were 3 months old, and the other two 1 month and 9 months old respectively) were admitted to a General Paediatrics Operating Unit. Their hospitalization period lasted between 3 and 11 days (mean 7.2 days). Three infants required oxygen therapy. No 
complications occurred. None of the mothers had been offered vaccination against pertussis during pregnancy.

The epidemiology of pertussis is changing in Italy as well as in other countries with high vaccination coverage. The disease is now increasing as an incidence in adolescents (and adults) who have lost their immune protection, and especially in new-born infants who have not started or completed the primary vaccination course yet. The data from the European Annual Epidemiological Report for 2016 report the highest notification rate in children $<1$ year $(73.6$ cases per 100,000 population), similar to that observed in 2015 (73.1). The vaccination of pregnant women against pertussis is considered an effective and available preventive option adopted for some years and in a systematic way in different international contexts. Also in Italy, in the 2017-2019 National Vaccination Plan there is a recommendation to recall in pregnancy the immunization from pertussis with the trivalent vaccine (whooping cough, tetanus and diphtheria). The period indicated is between the 27 th and the 36 th week, ideally around the 28th week. The vaccine strategy for pertussis in pregnant women is effective in reducing neonatal deaths due to the disease, the need for hospitalization and the severity of the infection in infants under the age of 3 months. The vaccine does not pose any risk for pregnant women and newborns.

From the description of our experience, of the 6 cases of hospitalized pertussis in just 3 months in a Pediatric department, 5 could be theoretically prevented by vaccination of the mother. The reported narrative experience testifies indirectly the resurgence of the cases of pertussis in a period when the newborn and the infant is most at risk to contract the disease and to have severe complications.

Despite the available evidence and the consolidated experiences carried out, the application of this strategy, in some industrialized countries (including Italy), although recommended, it results largely sub-optimal. Pertussis prevention in newborns and infants is an urgent measure of public health.

\section{P305 HEALTHY LIFE-STYLE FOR A HEALTHY CHILDHOOD - A PROGRAM OF EDUCATION FOR HEALTH IN CHILDREN}

'2nd Paediatric Clinic University of Medicine and Pharmacy "I Iuliu Hatieganu', Cluj-Napoca, Romania; ${ }^{2}$ Romanian Society of Social Paediatrics, Cluj-Napoca, Romania; ${ }^{3}$ Society of Physicians from Children and Youth Communities, Cluj-Napoca, Romania; ${ }^{4}$ Regina Maria Healthcare Network, Cluj-Napoca, Romania

\subsection{6/archdischild-2019-epa.654}

Introduction Non-communicable diseases (NCDs) account for $70 \%$ of global mortality and represent the leading cause of death and disability worldwide. In Romania, the cardiovascular diseases represent the first cause of mortality. Unhealthy behavior, malnutrition, both overweight or obesity, and physical inactivity are known risk factors for metabolic syndrome, which is involved as a cause for cardiovascular diseases in adults. The education for health in children is one of the most effective ways to reduce exposure to risk factors for NCDs and decrease the morbidity in the future adults.

"Healthy Life-style for a Healthy Childhood - A Healthy Child is A Happy Child!' is a program for education for health for children developed under the NCD Child Advocacy in Action Grant Program of the American Academy of Pediatrics. The goal of this program was to promote the healthy life-style for children of three schools from Cluj-Napoca, Romania. The objectives were education for healthy lifestyle in school-aged children (by publication of brochures, live courses about the importance of healthy lifestyle: nutrition, physical activity, healthy behaviors), promotion of healthy nutrition (including workshops with healthy cooking where children and their parents prepare different healthy meals) and promoting of physical activity (running events for children involved in the program).

The website of the program contains online courses accessible for children involved in the program, but also to other children of school-age, being produced in three languages (Romanian, Hungarian and English). There are modules about healthy life-style and nutrition, including quizzes, one collection of recipes for healthy meals where the children could promote theirs healthy recipes. The children involved in the project could participate in one running event and then one team represented the project in the main running event in our city.

Based on the experience gained in this project all the activities will continue during the following years with an extension in other schools from our city, for a larger number of school-aged children.

Conclusions The education for health, promoting the change of the life-style of our children with a healthier one is considered the most beneficial preventive action in order to have a healthy population in the future and to decrease the risk for metabolic syndrome and cardiovascular disorders.

\section{P306 ENVIRONMENTALLY DEPENDENT ENDOCRINOPATHIES IN THE CHILDREN FROM MOUNTAINOUS REGION}

${ }^{1}$ Olesya Horlenko*, ${ }^{1}$ Lyubov Pushkash, ${ }^{1}$ Vikroria Studeniak, Marianna Peresta 1 , ${ }^{1}$ Valeria Brych, ${ }^{1}$ Ivan Pushkash, ${ }^{2}$ Natalia Banadyga. 'Uzhhorod National University, Uzhhorod, Ukraine; ${ }^{2}$ Ternopil State Medical University, Ternopil, Ukraine

\subsection{6/archdischild-2019-epa.655}

Background The problem of iodine deficiency diseases (IDD) is relevant in connection with the significant occurrence of iodine deficiency among the population of many countries of the world, an increase in morbidity with a wide range of clinical manifestations and a marked tendency to increase the incidence and severity among children of all age groups.

Material and methods The research was conducted on the basis of the data of the preventive survey (187 children) living in the ecologically dependent biogeochemical endemic zone with iodine deficiency in the mountain region of the Transcarpathian region in the period of 2014-2015y.y.

Result Taking into account the morphometric characteristics of the thyroid gland in the chidren was found a slight difference between the standard values of unchanged parenchyma thyroid gland, the size of the gland was on $24.4 \%$ lower than the reference in the girls and on $27.5 \%$ in the boys in the patients with hypoplasia., The size of the thyroid gland of children with the hyperplasia was higher than the reference on $39.04 \%$ in the girls and on $31.8 \%$ in the boys. The data of the state of the thyroid system testify have differences in the levels of TSH - from $2.87 \pm 0.67 \mathrm{mO} / 1$ to $1.89 \pm 0.43$ $\mathrm{mO} / \mathrm{l}, \mathrm{p}=1.97 \times 10-8$, respectively, in groups in children with hyperplasia of the thyroid gland and with normal thyroid parenchyma. The levels of AT-TG are higher than the reference in children with thyroid hyperplasia and within the normal range in children with normal thyroid gland parenchyma, which suggests insignificant effects on the interference with the physiological process of T3 and T4 synthesis. The value 